\title{
Quality of Life of Patients with Free Flaps for Head and Neck Reconstruction after Tumor Resection
}

Wenlu $\mathrm{Li}^{*}$, Yanjie Yang and Juanfang Zhu

Department of Stomatolagy, The First Affiliated Hospital of Zhengzhou University, China

*Corresponding author: Wenlu Li, Department of Stomatolagy, The First Affiliated Hospital of Zhengzhou University, Zhengzhou, Henan 450052, China, Tel: 18842344771; E-mail: Iwldoctor@163.com

Received date: February 11, 2014, Accepted date: April 28, 2014, Published date: April 30, 2014

Copyright: () $2014 \mathrm{Li} \mathrm{W}$, et al. This is an open-access article distributed under the terms of the Creative Commons Attribution License, which permits unrestricted use, distribution, and reproduction in any medium, provided the original author and source are credited.

\begin{abstract}
Free flaps represent the first option for reconstruction of the head and neck oncologic defect. However, the assessment of patient satisfaction and quality of life (QOL) is also important. Therefore, assessment of the QOL in head and neck cancer patients has become an important aspect of postoperative care and even an aim of the therapeutic approach. Indeed, QOL has become an increasingly important outcome measure for patients undergoing treatment for a wide array of illnesses, and it reflects a patient's general sense of well-being.
\end{abstract}

Keywords: Free flaps; Head and neck reconstruction; Head and neck cancer

\section{Introduction}

Presently, it is generally acknowledged that free flaps transfer with microvascular anastomosis is the favored method for reconstruction after major head and neck cancer surgery. The success rates of free tissue transfer have steadily increased over this time span, such that free flap success rates are usually reported in excess of 95\% $[1,2]$. Successful reconstruction has often focused on the rate of survival of free flaps rather than on patients' quality of life. It is important to reconstruction with free flaps transfer to obtain a total or partial aesthetic and functional rehabilitation and a satisfying quality of life.

The terms "quality of life" and "health-related quality of life" were introduced in health-related literature in 1977. These terms are now widely used in various contexts to describe the impact of disease and health on personal experiences, whether physical, emotional, or social [3]. Assessment of the quality of life (QOL) provides information about the psychosocial well-being of patients and the effects of the disease and its treatment. Hence, it is an important tool for evaluating outcome in conjunction with mortality, morbidity, survival and recurrence rates.

\section{Results}

Recently, some papers have been published about the quality of life of the free flaps for head and neck reconstruction after tumor resection. QOL assessment in head neck cancer is critical not only to the evaluation of treatment options, but also to the development of rehabilitative services and patient education materials. Patients with head neck cancer report significant and persistent physical (such as: radionecrosis, mucositis, loss of taste, and dysphagia), functional (such as: pain, difficulty swallowing, voice impairment, and poor dental status), and psychosocial problems (such as: depression, disfigurement, social isolation, and delays returning to work). The expectation of the clinical outcome of reconstruction after operation for head neck cancer is regarded as an important factor.
Quality of Life questionnaire can better come to the status of the quality of life of patients. The relatively large number of questionnaires specific for diseases of the oral cavity reflects that there is no "gold standard". Because of the inconsistencies in design elements and the lack of unified reporting standards for studies testing QOL instruments, make it difficult to pool data in order to make general statements on QOL that will aid clinical decision making. One consistently different factor among studies is the choice of QOL instrument as evidenced by the differences in frequency of use of each available instrument by clinical studies in this review.

According to the Scientific Advisory Committee of the Medical Outcomes Trust (SAC-MOT) for the development and validation of health outcomes questionnaires [4] and other international guidelines, high-quality measures of QOL should be reliable, valid, and demonstrate responsiveness (the ability to detect change over time). Other characteristics to consider when evaluating measures include the conceptual and empirical basis for content generation, whether there is reasonable respondent and administrative burden, and whether the measure has been translated and validated for use in cross-cultural populations.

Previous study found that a select few of the dozens of available questionnaire are used consistently by researchers across studies. To be specific, site-specific questionnaires such as the UWQOL or the EORTC QLQ-H\&N35, tend to have a longer history of use since design and consequently have better reports of their properties, by comparison to instruments in other categories [5-7]. Nowadays, quality of life of patients with free flaps for head and neck reconstruction, the most application of the following questionnaire: Functional Living Index-Cancer (FLIC); Cancer Therapy-General (FACT-G), Head and Neck (FACT-HN) and University of Washington Quality of Life Scale (UW-QOL); EORTC QLQ-C30 (European Organization for Research and Treatment of Cancer Quality of Life Questionnaire version 3) and H\&N35 (Head and Neck Module); European Organization for Research and Treatment of Cancer Quality of Life Questionnaire-H\&N35 (EORTC H\&N35); University of Washington Quality of Life questionnaire(UW-QOL); University of Washington Quality of Life questionnaire(UW-QOL); 14-item Oral Health Impact Profile (OHIP-14) and the University of 
Citation: Li W, Yang Y, Zhu J (2014) Quality of Life of Patients with Free Flaps for Head and Neck Reconstruction after Tumor Resection. Anaplastology 3: 128. doi:10.4172/2161-1173.1000128

Page 2 of 2

Washington Quality of Life (UW-QOL); standardized FLZM questionnaire; Medical Outcomes Study-Short Form-36 (MOS SF-36) and the University of Washington Quality of Life (UW-QOL).

The most frequently utilized and thoroughly tested instruments in this category were: the EORTC QLQ-H\&N35, University of Washington QOL questionnaire (UW-QOL), the FACT-HN, and the University of Michigan Head and Neck QOL questionnaire. Other less utilized but well tested instruments include the: MD Anderson Symptom Inventory Head and Neck questionnaire, Head and Neck Cancer Inventory, and the Facial Skin Cancer Index (FSCI).

As the head and neck is responsible for many different functions, such as chewing of food, swallowing, production of saliva, speech, and breathing, and not least for interpersonal contacts such as kissing, a functional deficit leads to obvious changes in patients' QOL [8]. The expectation of the clinical outcome of reconstruction after operation for head neck cancer is regarded as an important factor [9]. Mari's study assessed swallowing during a one-year follow-up in 41 consecutive patients with large oral or oropharyngeal carcinoma. Their findings suggest that, one year after surgery, $86 \%$ of the patients ate regular masticated or soft food and swallowing problems should be routinely sought and patients rehabilitated during a sufficiently long follow-up with video fluorography regardless of the patient's perception of swallowing [10]. Hara et al. [11] used video fluoroscopy to assess tongue mobility and abnormalities of swallowing function, and their study show that patients who underwent anterior or posterior resection had greater decreases in tongue mobility than those who underwent medial resection.

\section{Conclusion}

In conclusion, head and neck cancers and its treatment (reconstruction with free flaps) have a disproportionate impact on all aspects of patient quality of life. QOL is a multi-dimensional construct of an individual's subjective assessment of the impact of an illness or treatment on his or her physical, psychological, social, and somatic functioning and general well-being. Given that QOL domains have been shown to predict survival among head neck cancer patients, it is not surprising that QOL has become an important treatment outcome in head neck cancer reconstruction with free flaps.

\section{References}

1. de Bree R, Rinaldo A, Genden EM, Suarez C, Rodrigo JP, et al. (2008) Modern reconstruction techniques for oral and pharyngeal defects after tumor resection. Eur Arch Otorhinolaryngol 265: 1-9.

2. Wei FC, Jain V, Celik N, Chen HC, Chuang DC, et al. (2002) Have we found an ideal soft- tissue flap? An experience with 672 anterolateral thigh flaps 109: 2219-2226.

3. MacEntee MI (2007) Quality of life as an indicator of oral health in older people. J Am Dent Assoc 138: 47-52.

4. Aaronson N, Alonso J, Burnam A, Lohr KN, Patrick DL, et al. (2002) Assessing health status and quality-of-life instruments: attributes and review criteria. Qual Life Res 11: 193-205.

5. Li W, Yang Y, Xu Z, Liu F, Cheng Y, et al. (2013) Assessment of quality of life of patients with oral cavity cancer who have had defects reconstructed with free anterolateral thigh perforator flaps. Br J Oral Maxillofac Surg 51: 497-501.

6. Li W, Xu Z, Liu F, Huang S, Dai W, et al. (2013) Vascularized free forearm flap versus free anterolateral thigh perforator flaps for reconstruction in patients with head and neck cancer: assessment of quality of life. Head Neck 35: 1808-1813.

7. Zhu J, Yang Y, Li W (2013) Assessment of quality of life and sociocultural aspects in patients with ameloblastoma after immediate mandibular reconstruction with a fibular free flap.Br J Oral Maxillofac Surg 51: 497-501.

8. Rogers SN, Scott J, Chakrabati A, Lowe D (2008) The patients' account of outcome following primary surgery for oral and oropharyngeal cancer using a "quality of life" questionnaire. Eur J Cancer Care 17: 182-188.

9. Chandu A, Smith AC, Rogers SN (2006) Health-related quality of life in oral cancer: a review. J Oral Maxillofac Surg 64: 495-502.

10. Markkanen-Leppänen M, Isotalo E, Mäkitie AA, Rorarius E, AskoSeljavaara S (2006) Swallowing after free-flap reconstruction in patients with oral and pharyngeal cancer. Oral Oncol 42: 501-509.

11. Hara I, Gellrich NC, Düker J, Schön R, Nilius M, et al. (2003) Evaluation of swallowing function after intraoral soft tissue reconstruction with microvascular free flaps. Int J Oral Maxillofac Surg 32: 593-599. 